\title{
Determination of Dissolution Profile and Bioaccessibility of Ketosteril Using an Advanced Gastrointestinal In Vitro Model
}

\author{
Edmundo Brito-de la Fuente*, Sébastien Secouard, Nadja Siegert, Francina Payeras Perelló, and \\ Crispulo Gallegos \\ Product and Process Engineering Center, Global Manufacturing Pharmaceuticals, Pharmaceuticals Division, Fresenius Kabi Deutschland GmbH, Bad \\ Homburg, Germany
}

e-mail: edmundo.brito@fresenius-kabi.com

\begin{abstract}
Ketosteril is an originator drug prescribed for nutrition therapy for patients with chronic kidney disease (CKD). Ketoanalogues (KAs) of amino acids are part of the active pharmaceutical ingredients in the Ketosteril film-coated tablets. The in vitro dissolution and gastrointestinal (GI) release of KAs available in Ketosteril were determined in this study using a dynamic multi-compartmental system, TIM-1. A water-fed mode of operation for TIM-1 was used to study the dissolution and bioaccessibility of KA during an experimental 5-hour test. Overall recovery (i.e., mass balance) and bioaccessibility as a function of time were the major outcome parameters. Based on eight experimental runs, the mean \pm SD percent recovery was $95.2 \pm 4.0$ (RSD: 4.2 ), showing high reproducibility for the dissolution of a solid dosage form in a highly complex in vitro model. The bioaccessible fraction (BioAcc) of KA was found to be highest at 2 and 3 hours. Furthermore, the total BioAcc was found to be higher for the jejunum (68.2\%-68.7\% of dosage) than the ileum (15.9\%$17.9 \%$ of dosage). The in vitro results of this study show that the release of Ketosteril KAs occurs at physiological relevant sites, mainly the jejunum, with its amino acid receptor for absorption, as it has been reported in clinical observations with humans. These bioaccessibility results may explain the clinical performance of Ketosteril reported in several clinical trials of patients with CKD with regards to postponing dialysis. Ketosteril in combination with a low protein diet has been proved to be a nutritionally safe therapy.
\end{abstract}

KEYWORDS: Ketosteril, dissolution profile, in vitro digestion, bioaccessibility, TIM-1 (TNO Intestinal Model)

\section{INTRODUCTION}

D issolution profiling of pharmaceutical oral dosage forms is often considered one of the foundations of research and development of new products. One of the challenges in this area is correlating in vitro drug release to their in vivo drug profiles, concept known as in vitro in vivo correlation (IVIVC). Drug performance can be significantly affected by dietary intake. Furthermore, changes in gastrointestinal physiology (e.g., stomach emptying time, luminal changes, intestine motility, and transit time variations, etc.) are variables involved in the final drug absorption rate and thus drug performance. Despite the understanding of these variables, many in vitro dissolution profiles of oral dosage forms used simplified systems that do not account for all aspects of human physiological conditions $(1,2)$. However, simplified models also represent useful alternatives for dissolution of oral drug products, as recently reviewed by Pentafragka et al. (3).

Conventional and compendial dissolution tests for pharmaceutical oral solid dosage forms using, for example, the United States Pharmacopoeia (USP) apparatus 1 and 2 , have been widely utilized for the purpose of quality control and batch-to-batch comparison. Dissolution test results from USP apparatus 1 and 2 may be appropriate to evaluate the dissolution of Biopharmaceutics Classification System (BCS) class I drugs (i.e., high soluble, high permeable), as originally proposed by Amidon et al. (4). However, USP apparatus 1 and 2 methods are unsuitable for assessment of bioaccessibility of low or poorly soluble drugs because dissolution of these drugs 
is highly dependent on physiological gastrointestinal (GI) variables some of which are listed above (4). Neither the USP, nor any other current compendial dissolution tests, take into consideration these GI variables. Consequently, these tests may not be considered as in vivo predictive methodologies of drug bioaccessibility and thus performance in the human body (5). However, biorelevant media, introduced to simulate the composition of luminal fluids, could be applied to those tests for the improved evaluation of formulation disintegration and drug release. To better understand in vitro drug dissolution profiles, absorption and bioaccessibility, the use of an apparatus that incorporates $\mathrm{Gl}$ physiology-related variables is of higher value.

A review on the non-conventional and non-compendial dissolution methods for pharmaceutical oral dosage forms was published by McAllister (6). In this review, the author highlighted the limitations of compendial methods and apparatus for drug dissolution profiling. Several systems that evolved from research in the nutritional field have been fully described by different authors as being closer to the human GI physiological conditions and, thus, more complete drug dissolution profiling and bioaccessibility data can be generated from these devices. A good overview on those systems addressing hydrodynamic conditions of the $\mathrm{Gl}$ has been provided by Pentafragka et al. (3). Some outstanding models that allow dissolution profiling of oral solid dosage forms under dynamic conditions are the fed stomach model (FSM) by Koziolek et al, the dynamic gastric model (DGM) from the Institute for Food Research (Norwich, UK), and the TIM-1 model from TNO Triskelion (7-11).

The most advanced, commercially available model is TIM1, a dynamic, computer-controlled, multicompartmental, in vitro $\mathrm{Gl}$ system $(10,11)$. In the TIM-1 model, it is possible to determine drug bioaccessibility, i.e., availability of active compounds for intestinal absorption $(12,13)$. Moreover, TIM-1 is already used by several companies and universities to determine drug and oral dosage form testing. The system simulates the conditions in the stomach and the small intestine, and it is validated for evaluating the digestion of foods, release of compounds, and availability for absorption of nutrients and drugs from various meals and dosage forms under fed and fasting states in adults $(11,14-21)$.

Furthermore, the numbers of TIM-1 studies on the evaluation of pharmaceutical dosage forms are increasing. For example, Abrahamsson et al. showed a food-induced delay in disintegration of immediate release tablets in vitro and in the stomach of dogs (22).
Cardot et al. describe that the TIM-1 mimics GI motility and the mechanical destructive force on drug release (23). The drug concentration can be measured in jejunal and ileal dialysis fluids, following its passive diffusion through the hollow-fiber membranes connected to the two compartments representing the jejunum and the ileum, respectively. Barker et al. pointed out that the TIM1 correctly predicts in vivo data for evaluation of drug product performance (20).

Ketosteril is an originator drug product prescribed for nutrition therapy in chronic kidney disease (CKD). Ketosteril tablets allow the intake of essential amino acids while minimising amino-nitrogen intake. Following absorption, the keto- and hydroxy-analogues (KA and HA, respectively), part of Ketosteril's active pharmaceutical ingredients (APIs), are transaminated to the corresponding essential amino acids by taking nitrogen from nonessential amino acids, thereby decreasing the formation of urea by reusing the amino group. Hence, the accumulation of uraemic toxins is reduced.

The use of Ketosteril in combination with a very low protein diet reduces nitrogen intake while preventing the deleterious consequences of inadequate dietary protein intake and malnutrition (24). The clinical benefits of a KA-supplemented, vegetarian, very low protein diet in patients with CKD have been recently proven by Garneata et al $(25,26)$. In this clinical trial, the authors observed a correction of metabolic abnormalities in those patients following the Ketosteril and the very low protein diet. Moreover, the authors were able to show a significant delay in entering dialysis for patients with CKD who were following the KA-supplemented (with Ketosteril) very low protein diet. This was a prospective, open, randomized controlled, single-center clinical study that involved more than 400 patients for 15 months.

As there is clinical evidence that CKD patients experience a significant delay in gastric emptying and that this might be related to a possible pathophysiological mechanism, it is then of high value to generate dissolution profiling and bioaccessibility data for Ketosteril, using in vitro techniques that are able to mimic, as close as possible, the $\mathrm{GI}$ conditions of CKD patients (27).

The small intestine is the principal site of protein and amino acids absorption in humans. Moreover, within the small intestine, there are regional variations in the absorptive capacities for protein digestion products (i.e., peptides and amino acids). The two groups of end products, namely amino acids and small peptides (i.e., dipeptides and tripeptides), are absorbed at different rates 
in different sections of the small intestine. The absorptive capacity for dipeptides and tripeptides is greater in the proximal small intestine (i.e., the duodenum) than in the distal small intestine (i.e., jejunum and ileum). On the other hand, in the case of amino acids, the absorptive capacity is greater in the distal small intestine than in the proximal small intestine $(28,29)$. It is the action of the membranebound peptidases in the brush-border membrane of the enterocyte that generates a major portion of the absorbable products, namely amino acids, dipeptides and tripeptides. Though these peptidases are present throughout the small intestine, their activities are much higher in the jejunum and ileum versus the duodenum. It is therefore conceivable that, as the luminal contents move along the intestine from the duodenum to the jejunum and then to the ileum, the rate of appearance of free amino acids in the lumen gradually increases whereas the luminal concentration of dipeptides and tripeptides gradually decreases (30).

In the present study, the dissolution of Ketosteril (the pharmaceutical dosage form of KAs and amino acids for CKD) has been studied in the TIM-1 model. The aim of this study was to establish a bioaccessibility profile of the APIs in KA. For this purpose, a complete validation of Ketosteril dissolution profiles in terms of overall recovery of KA and time-dependent bioaccessibility of KA was determined using TIM-1.

\section{MATERIALS AND METHODS}

\section{Test Product Composition and Excipients}

The commercially available, film-coated tablet formulation of Ketosteril (Fresenius Kabi, China) was used for this study. Each tablet contained the following KA calcium salts: $101 \mathrm{mg}$ of leucine (4-methyl-2-oxopentanoic acid); $86 \mathrm{mg}$ of valine (3-methyl-2-oxobutanoic acid); $68 \mathrm{mg}$ of phenylalanine (2-oxo-3-phenylpropanoic acid); and $67 \mathrm{mg}$ of DL-isoleucine (3-methyl-2-oxopentanoic acid). Additionally, each tablet contains $59 \mathrm{mg}$ of the HA calcium salt, DL-methionine (2-hydroxy-4-methylsulfanylbutanoic acid); $105 \mathrm{mg}$ of L-lysine acetate (75 mg of L-lysine); 53 $\mathrm{mg}$ of L-threonine; $23 \mathrm{mg}$ of L-tryptophan; $38 \mathrm{mg}$ of L-histidine; and $30 \mathrm{mg}$ of L-tyrosine. Total nitrogen and calcium content per tablet was 36 and $50 \mathrm{mg}$, respectively. Excipients include corn starch, crospovidone type A, talc, silica (colloidal anhydrous), vegetable magnesium stearate, macrogol 6000, quinoline yellow (E104), alkaline polymethacrylate, triacetin, titanium dioxide (E171), and povidone K 29-32 (24).

\section{Test Product Dosage}

To closely mimic the prescription recommendations (i.e., four to eight tablets during a meal), the dosage was set as five Ketosteril tablets per experiment. The product was stored at room temperature prior to the test. As this study aims to investigate the maximum release of APIs that can be reached from the studied test item without additional food matrix, no additional food was added to the five tablets used in each experimental run.

\section{In Vitro Testing TIM-1 design and mode of operation}

The multicompartmental, dynamic, computer-controlled model of the human upper GI tract, TIM-1 (TNO Triskelion, Netherlands), has been previously described by Minekus et al. and Verwei et al. $(10,11)$. The TIM-1 model consists of four serial compartments representing the stomach, duodenum, jejunum, and ileum, interconnected via peristaltic valves $(10,11)$. Each compartment is composed of two glass units with a flexible silicone inner wall, enclosing the lumen while the glass units are filled with water. The model simulates the physiological conditions of the stomach and small intestine including the dynamics of mixing (by periodically applying water pressure), gastric emptying and intestinal transit times (by water and gas pressure, monitored by pressure and level sensors, and according to a power exponential function), the gastric and intestinal $\mathrm{pH}$ values (monitored by $\mathrm{pH}$ sensors), body temperature (via jacket-vessel water), and the composition and activity of the secretion fluids (computer-controlled pumps). The curves describing the gastric emptying and ileal delivery were primarily described by Elashoff et al. (31).

Low molecular weight compounds are removed continuously via dialysis membrane systems connected to the jejunal and ileal compartments of the system. This allows quantification of the bioaccessibility, i.e., the amount of digested product or drug substance in solution and available for absorption $(12,17,32)$. The use of these membrane systems means that in vivo processes such as active transport, efflux, and intestinal wall metabolism are not modelled mechanistically by the system.

In this study, the TIM-1 system was operated in water mode, using dialysis units (SUREFLUX-7L Dialyzer, Nipro Corporation, Japan) to mimic absorption of dissolved KAs (alternative available hardware mode is "lipid" using lipid filters). The TIM-1 incorporates two of these dialysis units; one in the jejunum and one in the ileum compartments. 
The dialyzed fluid was flushed at a preset rate of $10 \mathrm{~mL} /$ $\min$.

All experiments in the present study were performed under fed conditions (alternative condition is e.g., fasted with a quicker gastric emptying rate and lower initial $\mathrm{pH}$ value or any user defined condition), where media and device parameters mimic a fed state (i.e., digestion of a meal) with a stomach half emptying time of $80 \mathrm{~min}$, following the Elashoff equation, and a gradually lowered $\mathrm{pH}$, as described in Table 1 (31). This setting was chosen to closely mimic the conditions of in vivo administration and to account for delayed gastric emptying, as in patients suffering from CKD, the intended patient group for Ketosteril (27).

Table 1. Parameters of In Vitro GI Conditions in Fed State

\begin{tabular}{|c|c|}
\hline $\begin{array}{l}\text { Parameter of In Vitro GI } \\
\text { Conditions }\end{array}$ & Fed State \\
\hline Stomach Compartment & $\begin{array}{l}0 \mathrm{~h}, \mathrm{pH} 5.5 ; 0.5 \mathrm{~h}, \mathrm{pH} 4.5 ; 1 \mathrm{~h}, \mathrm{pH} 3 ; 2 \mathrm{~h}, \mathrm{pH} \\
2 ; 2.5 \mathrm{~h}, \mathrm{pH} 1.7 ; 5 \mathrm{~h}, \mathrm{pH} 1.7\end{array}$ \\
\hline Start residues & $\begin{array}{l}\text { Meal: } 5 \text { untreated tablets }(4.0 \pm 0.1 \mathrm{~g}) \text {, } \\
\text { completed to } 70.0 \mathrm{~g} \text { with deionized water } \\
\text { Stomach secretion: } 153.3 \mathrm{~g} \text { of } \mathrm{GES}, 7.7 \mathrm{~g} \text { of } \\
\text { enzyme solution, } 69.0 \mathrm{~g} \text { of deionized water, } \\
\text { and } 11.0 \mathrm{mg} \text { of alpha amylase (A6380). }\end{array}$ \\
\hline Secretion rate & $\begin{array}{l}0.25 \mathrm{~mL} / \mathrm{min} \text { of enzyme solution } \\
0.25 \mathrm{~mL} / \mathrm{min} \text { of deionized water }{ }^{1}\end{array}$ \\
\hline $\begin{array}{l}\text { Time of half emptying } \\
\left(t_{1 / 2}\right)\end{array}$ & $80 \mathrm{~min}$ \\
\hline Duodenal Compartment & Maintained at $\mathrm{pH} 5.9$ \\
\hline Start residues & $\begin{array}{l}\text { Filled to the level sensor with duodenal } \\
\text { fluid solution }(\sim 60 \mathrm{~g})\end{array}$ \\
\hline Secretion rates & $\begin{array}{l}0.50 \mathrm{~mL} / \mathrm{min} \text { of bile solution } \\
0.25 \mathrm{~mL} / \mathrm{min} \text { of pancreatic solution } \\
0.25 \mathrm{~mL} / \mathrm{min} \text { of SIES }\end{array}$ \\
\hline Jejunal Compartment & Maintained at $\mathrm{pH} 6.5^{3}$ \\
\hline Start residues & Filled to the level sensor with SIES ( $90 \mathrm{~g}$ ) \\
\hline Dialysis rate & $10 \mathrm{~mL} / \mathrm{min}$ of SIES \\
\hline Ileal Compartment & Maintained at $\mathrm{pH} 7.4^{3}$ \\
\hline Start residues & Filled to the level sensor with SIES ( $90 \mathrm{~g})$ \\
\hline Dialysis rate & $10 \mathrm{~mL} / \mathrm{min}$ of SIES \\
\hline
\end{tabular}

${ }^{1}$ Substituted with $1 \mathrm{M} \mathrm{HCl}$ for $\mathrm{pH}$ adjustment, ${ }^{2}$ Substituted with $1 \mathrm{M} \mathrm{NaHCO}_{3}$ for $\mathrm{pH}$ adjustment, ${ }^{3}$ Secretion of $1 \mathrm{M} \mathrm{NaHCO}_{3}$ for $\mathrm{pH}$ adjustment.

GI: Gastrointestinal; GES: Gastric electrolyte solution; SIES: Small intestine electrolyte solution; $\mathrm{HCl}$, hydrochloric acid; $\mathrm{NaHCO}_{3}$, sodium bicarbonate.

\section{TIM-1 media}

The TIM-1 system was loaded with various reagents, mixed in specified ratios as described below. Some were derived from concentrated stock solutions, whereas all working solutions were prepared on the day of use.
All solutions were made with deionized water unless specified.

The solutions were:

- $1 \mathrm{M}$ sodium bicarbonate, used in the small intestine compartments

- $1 \mathrm{M}$ hydrochloric acid, used in the stomach compartment

- $1 \mathrm{M}$ sodium acetate buffer, $\mathrm{pH} 5$

- Gastric electrolyte solution (GES): $6.3 \mathrm{~g} / \mathrm{L}$ sodium chloride, $2.2 \mathrm{~g} / \mathrm{L}$ potassium chloride, $0.3 \mathrm{~g} / \mathrm{L}$ calcium chloride dihydrate

- Small intestine electrolyte solution (SIES): $5.4 \mathrm{~g} / \mathrm{L}$ sodium chloride, $0.65 \mathrm{~g} / \mathrm{L}$ potassium chloride, and $0.33 \mathrm{~g} / \mathrm{L}$ calcium chloride dihydrate

- Pancreatin solution: $65.4 \mathrm{~g} / \mathrm{L}$ pancreatin powder (Pancrex-Vet, Pfizer, Inc., USA) in deionized water

- Enzyme solution: $150 \mathrm{~g}$ of GES solution $\mathrm{pH}$ adjusted to $\mathrm{pH} 5$ using $1 \mathrm{M}$ sodium acetate buffer, containing 5,625 Units lipase (Lipase F-AP15 [Rhizopus oryzae], Amano Enzyme, Inc., Nagoya, Japan), 90,000 Units pepsin (P7012, SigmaAldrich Corp., Steinheim, Germany)

- Bile solution: $38.5 \mathrm{~g} / \mathrm{L}$ bile extract powder (porcine) in deionized water

- Duodenal fluid solution: $15 \mathrm{~g}$ of SIES solution, 15 $\mathrm{g}$ of pancreatin solution, $30 \mathrm{~g}$ of bile solution, and $1 \mathrm{~mL}$ of trypsin solution containing $2 \mathrm{mg}$ trypsin (T9201).

All parameters of the system were adjusted to simulate the fed state conditions (Table 1). Unless otherwise stated, reagents were supplied by Sigma-Aldrich Chemie $\mathrm{GmbH}$ (Schnelldorf, Germany). For each experiment, the secretion products (e.g., gastric juice with enzymes, electrolytes, dialysis liquids, bile, and pancreatic juice) were freshly prepared, the $\mathrm{pH}$ electrodes calibrated, and the dialysis units replaced.

\section{TIM-1 sampling and ketoanalogues assay}

Samples were collected every hour for 5 hours from jejunum and ileal dialysates (analogous to dissolved KA) combined with absorbance fractions. Samples were also collected every 2.5 hours from the outlet of the ileal compartment (analogous to KA entering the colon and assumed not to be available for absorption). At the 
end of the experiment, the device was stopped and all residues from stomach to ileum were collected to extract any remaining KA. Samples of stomach and duodenum residues, as well as jejunum and ileum residues, were combined prior to analysis, respectively. All samples were stored at $-20^{\circ} \mathrm{C}$ until KA determination.

\section{Ketoanalogue determination}

All samples were analyzed for KA content by highperformance liquid chromatography (HPLC) (Alliance 2695 HPLC Separations Module [Waters Corp., USA] and Aminex HPX-87H, $300 \times 7.8 \mathrm{~mm}$ columns [Bio-Rad Laboratories, Inc., USA]) at room temperature $\left(20-22^{\circ} \mathrm{C}\right)$, using $0.025 \mathrm{M} 95 \%$ sulphuric acid (Merck KGaA, Germany) as the mobile phase, at a flow rate of $0.45 \mathrm{~mL} / \mathrm{min}$ for 40 min. Quantification was determined against standard reference solutions at $205 \mathrm{~nm}$ (Photodiode Array Detector UV/Vis 2489 [Waters Corp., USA]).

\section{Data Presentation and Statistical Analysis}

One major output parameter from the TIM-1 experiments was the bioaccessible fraction (BioAcc) of KA, which is equal to the percentage of KA absorbed by dialysis in the jejunum and ileum divided by the intake (tested dosage).

The BioAcc was compared at 1, 2, 3, 4, and 5 hours after the start of the experiment. The cumulative value was also compared.

The recovery of KA in the TIM-1 experiments, another major outcome parameter, was determined as the sum of KAs obtained from the jejunal and ileal dialysates (including absorbance), the output of the ileal compartment (ileum efflux), and residue samples at the end of the experiment for all compartments.

The relative percent recovery was obtained by dividing the recovery by the tested dosage.

Repeatability was evaluated by obtaining replicate measurements of the experimental runs. It was then estimated by calculating the relative standard deviation (RSD) of the multiple consecutive experiments. To study the TIM-1 model repeatability, 11 repetitive experiments were performed for two batches (batch A, $n=8$; batch $\mathrm{B}$, $n=3$ ) of Ketosteril. This approach allows the validation of the proposed protocol to determine the BioAcc.

\section{RESULTS AND DISCUSSION}

\section{Relative Recovery}

The relative recovery in the TIM-1 system for two Ketosteril batches is presented in Table 2. The range of relative recovery for experiments included in this study was $85 \%-100 \%$. From all experimental runs performed in this study using the TIM-1, 85\% passed this criterion. The relative mean \pm SD percent recovery for batch $A$ and B was $95.2 \pm 4.0 \%$ (RSD: 4.2 ) and $94.2 \pm 6.3 \%$ (RSD: 6.7 ), respectively. The unrecovered material $(<5 \%)$ is due to residues remaining in the entire model (e.g., prefilters, level sensors, $\mathrm{pH}$ electrodes) that cannot be made available for analysis. The relative recovery values show a very high overall recovery, as well as a high reproducibility for the determination of KA dissolution in the complex TIM-1 in vitro model. The high relative recovery shown in Table 2, which is a mass balance of the KA in the experimental runs, clearly suggests that this complex in vitro technique is reliable.

Table 2. Relative Recovery of Alpha Ketoanalogues

\begin{tabular}{|c|c|}
\hline Run & Relative Recovery (\%) \\
\hline A-1 & 99.1 \\
\hline A-2 & 97.0 \\
\hline A-3 & 93.4 \\
\hline A-4 & 95.5 \\
\hline A-5 & 97.3 \\
\hline A-6 & 99.6 \\
\hline A-7 & 91.6 \\
\hline A-8 & 88.0 \\
\hline B-1 & 97.2 \\
\hline B-2 & 87.0 \\
\hline B-3 & 98.5 \\
\hline
\end{tabular}

Mean $\pm S D$ (RSD) was $95.2 \pm 4.0$ (4.2) for batch $A(A-1-A-7)$ and $94.2 \pm 6.3$ (6.7) for batch $B(B-1-B-3)$., $R S D$, relative standard deviation.

\section{Ketosteril Dissolution - Visual Observations}

The gastric $\mathrm{pH}$ values were set to mimic data observed in vivo after ingestion of a meal (fed conditions). These values are continuously adjusted automatically by adding water or hydrochloric acid to the gastric compartment. As shown in Figure 1, upon introduction of five tablets in the gastric compartment of the TIM-1 apparatus, the solid dosage form remained visually intact during the first 10 minutes of an experiment. Subsequently, tablets disintegrated and dissolved progressively. After approximately 2 hours, no remaining tablet residues were observed.

\section{Distribution of Ketoanalogues from Ketosteril in the TIM-1 Compartments}

The amount of KA as determined in the specific compartments and residual samples is shown in Figure 2. No significant differences were observed for the two 
batches studied. The KA BioAcc obtained for the jejunum and ileum compartments was $68.2 \%-68.7 \%$ and $15.9 \%-$ $17.9 \%$, respectively, for both batches. Therefore, the highest amount of KA was found in the jejunum BioAcc. As expected, most of the pharmaceutical dosage dissolved in the upper $\mathrm{Gl}$ tract. This fraction can be considered available for absorption (i.e., bioaccessible) in the human gut. The total KA BioAcc is the sum of the jejunum and ileum BioAcc.

In contrast, the amount of KA in the ileum efflux is considered unavailable in the small intestine (Fig. 2). However, as the content is theoretically delivered to the colon, bioaccessibility of KA from the ileum efflux into the colon compartment is most likely. The amount of $\mathrm{KA}$ remaining in the compartments at the end of the experimental runs (i.e., stomach and duodenum residue, and the jejunum and ileum residue) was less than $5 \%$ for both batches. The amount of KA obtained in the residue fractions can be considered as potentially available for bio-accessibility; however, KA remaining in stomach and duodenum had no access to the site of dialyzation in the TIM-1 apparatus.

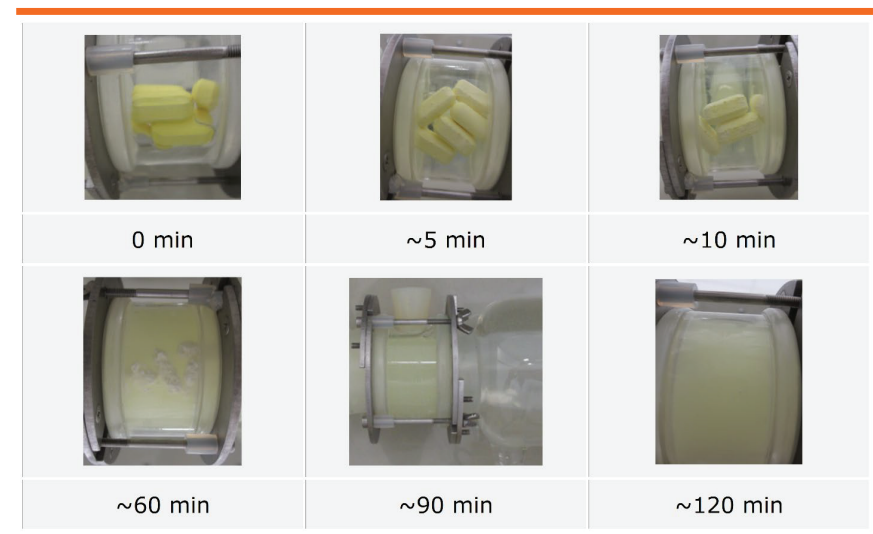

Figure 1. Disintegration of Ketosteril tablets in the gastric compartment over time.

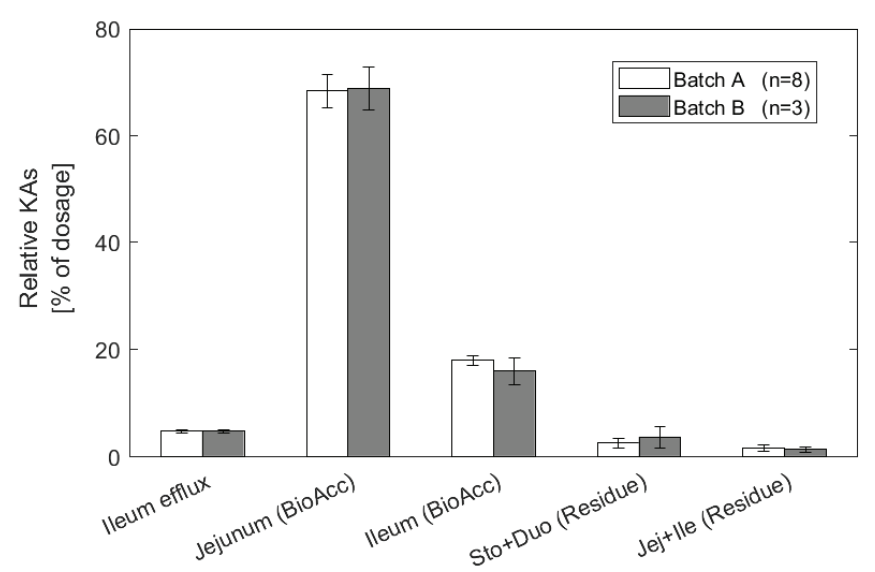

Figure 2. Relative $K A$ (mean $\pm S D$ ) in different compartments and samples for batch $A$ and B. KA: ketoanalogue; Sto, stomach; Duo, duodenum, Jej, jejunum, Ile, ileum, BioAcc, bioaccessibility fraction.

\section{Time-Dependent Bioaccessibility of Ketoanalogues from Ketosteril}

In the present study, bioaccessibility is expressed as relative BioAcc (related to the tested dosage). As described previously, the kinetic profile of KA bioaccessibility was measured in samples collected hourly from jejunal and ileal dialysates.

Figure 3 shows the total cumulative relative KA BioAcc as a function of time in the TIM-1 system. Figure $3 \mathrm{~A}$ shows the total BioAcc as a sum of the jejunum and ileum BioAcc, and Figure 3B shows the BioAcc from the jejunum and ileum separately. Figure 4 depicts the BioAcc for the specific experimental hours.
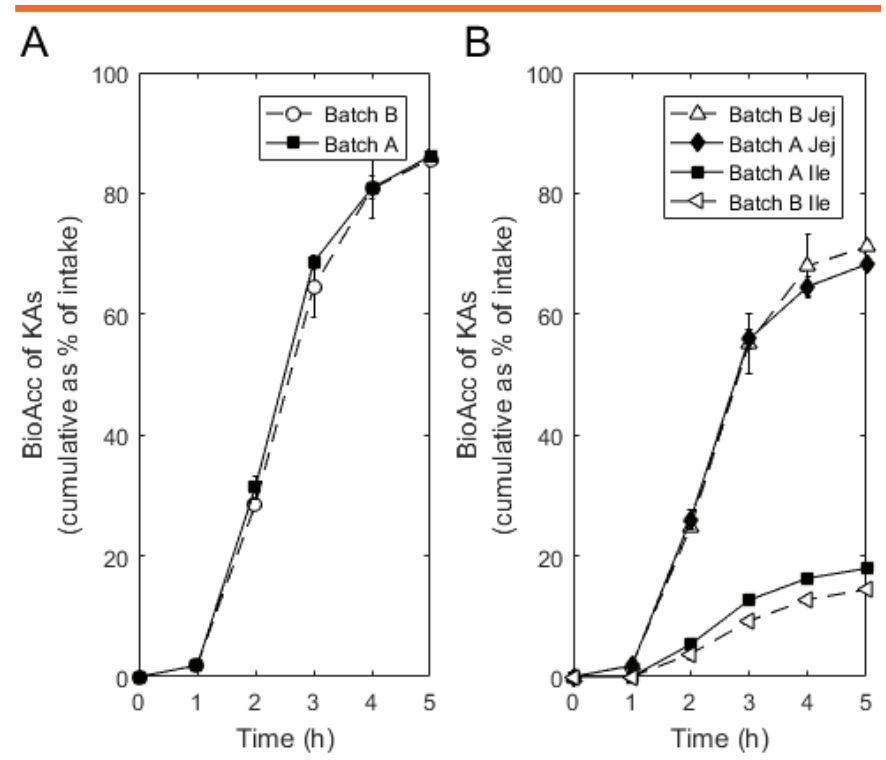

Figure 3. A. Cumulative, relative BioAcc of $K A$ s as sum of jejunum (Jej) and ileum (Ile). B. Cumulative, relative BioAcc of $K A$ s, individually for jejunal and ileum. Results are expressed as mean $\pm S D(n=8$ for batch $A, n=3$ for batch B). BioAcc: bioaccessible fraction; KA: ketoanalogue.

As shown in Figure $3 \mathrm{~A}$, no differences in the relative $\mathrm{KA}$ BioAcc were found for batches $A$ and $B$. Upon introduction of the oral solid dosage forms into the gastric compartment of the TIM-1 system, disintegration and dissolution of the solid dosage form occurred slowly (Fig. 1). This visual observation is reflected in a low BioAcc of less than $5 \%$ for jejunum and ileum in the first experimental hour (Fig. 3).

As expected, the BioAcc is very low in the first experimental hour, due to the dosage form as well as the technical setup. While the dosage form requires a certain time to disintegrate in the stomach, at least to particles sizes that can be transported into the small intestine, the technical aspect of the TIM-1 furthermore only allows a controlled amount of lumen fluid to enter from the stomach into the duodenum and the jejunum (first place of dialysation) for specific time intervals (following the 
Elashoff equation) (31). Therefore, only starting from the time point when KA are released from its dosage form and are then gradually transported with the lumen fluid to the site of action (jejunum, ileum), they can be dialyzed and thus found in the BioAcc.

A sharp increase in KA BioAcc occurs between 1 and 3 hours after the start of the experiment (Fig. 3), with the release being maximal between the second and third hours for both the jejunum and ileum dialysate fractions (Fig. 4). The cumulative BioAcc that was determined in the jejunum is higher than the cumulative BioAcc that was determined in the ileum for all the experiments (Fig. 3B). Solubility of the API is required to enable the transport through the dialysis units into the BioAcc. The solubilization must be assumed due to the high presence of API in the BioAcc of the jejunum. Therefore, KAs are already available for absorption in the jejunum compartment, and no further disintegration and solubilization of KA, as present in Ketosteril, seems to be required in the small intestine. Additionally, the release of KAs in the jejunum BioAcc was similar between the first and second and the second and third hours, while the release of KAs was markedly increased in the ileum BioAcc from the second to third hours (Fig. 3B). That difference could be explained by the impact of transport time, which is longer for reaching the ileum than for the jejunum. These results are in good accordance with the visual observations in the stomach, which showed that the disintegration of the tablets was completed in the stomach after 2 hours. The dissolved KA are transported within the lumen fluid from the stomach via the duodenum into jejunum and ileum, where they are gradually dialyzed.

As shown in Figure $3 \mathrm{~A}$, after 2 hours of digestion, the KA BioAcc was $28.8 \%$. This value is lower than the value reported by Framroze et al., who found $67 \%$ bioaccessibility for amino acids from salmon protein hydrolysates after 2 hours (33). This difference could be explained due to the disintegration time required for Ketosteril, which is not necessarily the same for protein hydrolysates.

As shown in Figure 3B, after approximately 3 hours, the KA BioAcc decreased gradually, both in the jejunum and ileum, until the end of the experiment, reaching final cumulative values of $86.1 \%$ and $84.6 \%$ for batch $A$ and B, respectively (Fig 3A). Nevertheless, after 5 hours of digestion, the cumulative KA BioAcc did not reach a steady state, suggesting a remaining fraction of KA in the TIM-1.
The results shown in Figures $2-4$ are in line with what is known regarding amino acids absorption in the human gut. Even though the process of protein digestion in the Gl tract is initiated by pepsin in the stomach, absorption of the digestion product is negligible at this site. The small intestine is the principal site of protein and amino acids absorption. Results shown in Figures 2-4 confirm that Ketosteril APIs are bioaccesible at physiological relevant sites for amino acids, mainly in the jejunum and ileum.

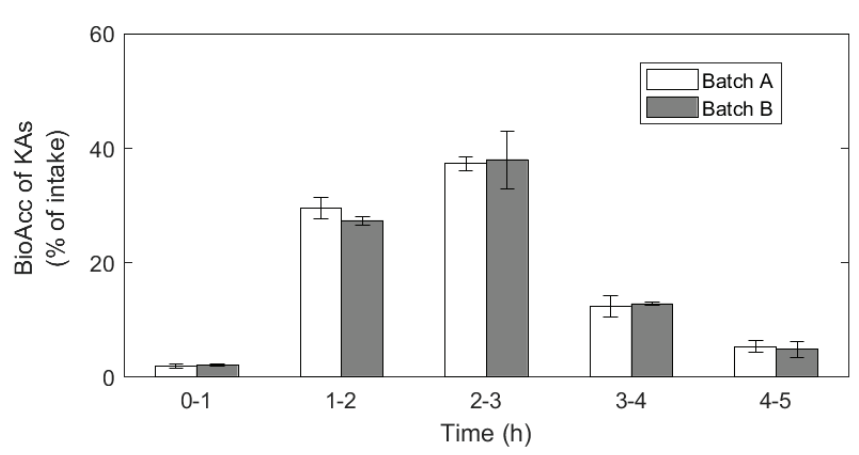

Figure 4. KA BioAcc (mean $\pm S D)$ over time. KA: ketoanalogue; BioAcc: bioaccessible fraction.

\section{CONCLUSIONS}

The in vitro dissolution Ketosteril data from this work, using a complex dynamic digestion system like TIM-1, with physiological conditions close to those found in patients with CKD, clearly suggest that, in spite of the operational complexity of digestion system, TIM-1 can provide highly reproducible results and thus reliable in vitro bioaccessibility data $(24,27)$.

In the present study, the bioaccessibility of Ketosteril was expressed as the amount of KA that was dialyzed from the jejunal and ileal compartments of the TIM-1 apparatus. It could be shown that the dynamic multi-compartmental TIM-1 model, which currently allows the closest simulation of in vivo physiological processes occurring within the human stomach and small intestine, could be successfully applied for the dissolution of Ketosteril.

The results from this work agree with what is physiologically known regarding the absorption of amino acids in humans. Amino acids are preferentially absorbed in the distal small intestine (i.e., the jejunum and ileum). The in vitro results from this work showed that the KAs of amino acids present in Ketosteril are mostly bioaccessible in the jejunum, followed by the ileum. This bioaccessibility profile may be used to explain the clinical performance of Ketosteril proving to have a potential to postponing dialysis needs in patients with CKD. Ketosteril 
in combination with a low protein diet has been proved to be a nutritionally safe therapy $(25,26)$.

The dissolution profile of $K A$, as determined with the TIM-1 system, mimics and predicts the in vivo situation to a far higher extent than commonly used classic dissolution methods (USP apparatus 1 and 2). The TIM1 system can be considered as a very useful tool for in vitro bioaccessibility studies and for the development of an IVIVC for well-established drugs in the market, as well as for new oral solid forms $(16,20)$.

\section{ACKNOWLEDGEMENT}

The authors thank their quality control colleagues from Fresenius Kabi (Portugal) for supporting the chemical analysis of KAs in the samples taken during this study, as well as colleagues from Fresenius Kabi (China) for providing several Ketosteril batches for use in research programs with the TIM-1 system.

\section{CONFLICT OF INTEREST}

The authors declared no conflicts of interest related to this article.

\section{REFERENCES}

1. Waiver of In Vivo Bioavailability and Bioequivalence Studies for Immediate-Release Solid Oral Dosage Forms Based on a Biopharmaceutics Classification System; Guidance for Industry; U.S. Department of Health and Human Services, Food and Drug Administration, Center for Drug Evaluation and Research (CDER), U.S. Government Printing Office: Washington, DC, 2000.

2. Guideline on the Investigation of Bioequivalence; CPMP/EWP/ QWP/1401/98 Rev. 1; Committee for Medicinal Products for Human Use (CHMP), European Medicines Agency: London, 2010.

3. Pentafragka, C.; Symillides, M.; McAllister, M.; Dressman, J.; Vertzoni, M.; Reppas, C. The impact of food intake on the luminal environment and performance of oral drug products with a view to in vitro and in silico simulations: a PEARRL review. J. Pharm. Pharmacol. 2019, 71, 557-580. DOI: 10.1111/jphp.12999.

4. Amidon, G. L.; Lennernäs, H.; Shah, V. P.; Crison, J. R. A theoretical basis for a biopharmaceutic drug classification: the correlation of in vitro drug product dissolution and in vivo bioavailability. Pharm. Res. 1995, 12, 413-420. DOI: 10.1208/s12248-0149620-9.

5. Matsui, K.; Tsume, Y.; Amidon, G. E.; Amidon, G. L. In vitro dissolution of fluconazole and dipyridamole in gastrointestinal simulator (GIS), predicting in vivo dissolution and drug-drug interaction caused by acid-reducing agents. Mol. Pharm. 2015, 12, 2418-2428. DOI: 10.1021/acs.molpharmaceut.5b00135.

6. McAllister, M. Dynamic dissolution: a step closer to predictive dissolution testing? Mol. Pharm. 2010, 7, 1374-1387. DOI: 10.1021/mp1001203.
7. Koziolek, M.; Goerke, K.; Neumann, M.; Garbacz, G.; Weitschies, W. Development of a bio-relevant dissolution test device simulating mechanical aspects present in the fed stomach. Eur. J. Pharm. Sci. 2014, 57, 250-256. DOI: 10.1016/j.ejps.2013.09.004.

8. Wickham, M. J. S.; Faulks, R. M.; Mann, J.; Mandalari, G. The design, operation and application of a dynamic gastric model. Dissolution Technol. 2012, 3, 15-22. DOI: 10.14227/ DT190312P15.

9. Mann, J. C.; Pygall, S. R. A formulation case study comparing the dynamic gastric model (DGM) with conventional dissolution methods. Dissolution Technol. 2012, 4, 14-19. DOI: 10.14227/ DT190412P14.

10. Minekus, M.; Marteau, P.; Havenaar, R.; Huis in't Veld, J. H. J. A multicompartmental dynamic computer-controlled model simulating the stomach and small intestine. Atla. 1995, 23, 197209.

11. Verwei, M.; Arkbage, K.; Havenaar, R.; van den Berg, H.; Witthoft, C.; Schaafsma, G. Folic acid and 5-methyltetrahydrofolate in fortified milk are bioaccessible as determined in a dynamic in vitro gastrointestinal model. J. Nutr. 2003, 133, 2377-2383. DOI: 10.1093/jn/133.7.2377.

12. Fernandez-Garcia, E.; Carvajal-Lerida, I.; Perez-Galvez, A. In vitro bioaccessibility assessment as a prediction tool of nutritional efficiency. Nutr. Res. 2009, 29, 751-760. DOI: 10.1016/j. nutres.2009.09.016.

13. Ribnicky, D. M.; Roopchand, D. E.; Oren, A.; Grace. M.; Poulev. A.; Lila, M. A.; Havenaar, R.; Raskin, I. Effects of a high fat meal matrix and protein complexation on the bioaccessibility of blueberry anthocyanins using the TNO gastrointestinal model (TIM-1). Food Chem. 2014, 142, 349-357. DOI: 10.1016/j. foodchem.2013.07.073.

14. Larsson, M.; Minekus, M.; Havenaar, R. Estimation of the bioavailability of iron and phosphorus in cereals using a dynamic in vitro gastrointestinal model. J. Sci. Food Agric. 1999, 73, 99-106. DOI: 10.1002/(SICI)1097-0010(199705)74:1<99::AIDJSFA775>3.0.CO;2-G.

15. Naylor, T. A.; Connolly, P. C.; Martini, L. G.; Elder, D. P.; Minekus, M.; Havenaar, R.; Zeijdner, E. Use of a gastrointestinal model and Gastroplus for the prediction of in vivo performance. Ind. Pharm. 2006, 12, 9-12.

16. Souliman, S.; Blanquet, S.; Beyssac, E.; Cardot, J. M. A level in vitro/in vivo correlation in fasted and fed states using different methods: Applied to solid immediate release oral dosage form. Eur. J. Pharm. 2006, 27, 72-79. DOI: 10.1016/j.ejps.2005.08.006.

17. Souliman, S.; Beyssac, E.; Cardot, J. M.; Denis, S.; Alric, M. Investigation of the biopharmaceutical behavior of theophylline hydrophilic matrix tablets using USP methods and an artificial digestive system. Drug Dev. Ind. Pharm. 2007, 33, 475-483. DOI: 10.1080/03639040601128654.

18. Mateo, A. N.; Van den Berg, R.; Havenaar, R.; Bast, A.; Haenen, G. Bioavailability of ferulic acid is determined by its bio-accessibility. J. Cereal Sci. 2009, 49, 296-300. DOI: 10.1016/j.jcs.2008.12.001. 
19. Brouwers, J.; Anneveld, B.; Goudappel. G.J.; Duchateau, G.; Annaert, P.; Augustijns, P.; Zeijdner, E. Food-dependent disintegration of immediate release fosamprenavir tablets: In vitro evaluation using magnetic resonance imaging and a dynamic gastrointestinal system. Eur. J. Pharm. Biopharm. 2011, 77, 313-319. DOI: 10.1016/j.ejpb.2010.10.009.

20. Barker, R.; Abrahamsson, B.; Kruusmägi, M. Application and validation of an advanced gastro-intestinal in vitro-model for evaluation of drug product performance in pharmaceutical development. J. Pharma. Sci. 2014, 103, 3704-3712. DOI: 10.1002/jps.24177.

21. Denis, S.; Sayd, T.; Georges, A.; Chambon, C.; Chalancon, S.; Santé-Lhoutellier, V.; Blanquet-Diot, S. Digestion of cooked meat proteins is slightly affected by age as assessed using the dynamic gastrointestinal TIM model and mass spectrometry. Food Funct. 2016, 7, 2682-2691. DOI: 10.1039/c6fo00120c.

22. Abrahamsson, B.; Albery, T.; Eriksson, A.; Gustafsson, I.; Sjöberg, M.; Food effects on tablet disintegration. Eur. J. Pharm. Sci. 2004, 22, 165-172. DOI: 10.1016/j.ejps.2004.03.004.

23. Cardot, J.M.; Beyssac, E.; Alric, M. In vitro-in vivo correlation: importance of dissolution in IVIVC. Dissolution Technol. 2007, 14, 15-19. DOI: 10.14227/DT140107P15.

24. Ketosteri ${ }^{\circledR}$ film coated tablets, Specific Product Characteristics (SmPC). Fresenius-Kabi Deutschland, Bad Homburg, 2014.

25. Garneata, L.; Stancu, A.; Dragomir, D.; Stefan, D.; Mircescu, L. Ketoanalogue-supplemented vegetarian very low-protein diet and CKD progression. J. Am. Soc. Nephrol. 2016, 27, 1-13. DOI: 10.1681/ASN.2015040369.

26. Yen, C.-L.; Tu, K.-H.; Lin, M.-S.; Chang, S.-W.; Fan, P.-C.; Hsiao, C.-C.; Chen, C.-Y.; Hsu, H.-H.; Tian, Y.-C.; Chang, C.-H. Does a supplemental low-protein diet decrease mortality and adverse events after commencing dialysis? A nationwide cohort study. Nutrients. 2018, 10, E1035. DOI: 10.3390/nu10081035.

27. Strid, H.; Simrén, M.; Stotzer, P. O.; Abrahamsson, H.; Björnsson, E. S. Delay in gastric emptying in patients with chronic renal failure. Scand. J. Gastroenterol. 2004, 39, 516-520. DOI: 10.1080/00365520410004505.

28. Matthews, D.M.; Cramption, R.F.; Lis, M.T. Sites of maximal intestinal absorption capacity for amino acids and peptides: evidence for an independent peptide uptake system or systems. J. Clin. Pathol. 1971, 24, 882-883. DOI: 10.1136/jcp.24.9.882-b.

29. Silk, D. B. A.; Webb, J. P. W.; Lane, A. E.; Clark, M. L.; Dawson, A. M. Functional differentiation of human jejunum and ileum: a comparison of the handling of glucose, peptides and amino acids. Gut. 1974, 15, 444-449. DOI: 10.1136/gut.15.6.444.

30. Ganapathy, V.; Gupta, N.; Martindale, G. Protein digestion and absorption. In: Physiology of the Gastrointestinal Tract, 4th ed; Elsevier, 2006, pp 1667-1692. DOI: 10.1016/B978-0120883943/50068-4.

31. Elashoff, J. D.; Reedy, T. J.; Meyer, J. M. Analysis of gastric emptying data. Gastroenterology 1982, 83, 1306-1312. DOI: 10.1016/S0016-5085(82)80145-5.

32. Blanquet, S.; Zeijdner, E.; Beyssac, E.; Meunier, J. P.; Denis, S.; Hayenaar, R.; Alric, M. A dynamic artificial gastrointestinal system for studying the behavior of orally administered drug dosage forms under various physiological conditions. Pharm. Res. 2004, 21, 585-591. DOI: 10.1023/B:PHAM.0000022404.7 0478.4b.

33. Framroze, B.; Savard, P.; Gagnon, D.; Richard, V.; Gauthier, S. F. Comparison of nitrogen bioaccessibility from salmon and whey protein hydrolysates using a human gastrointestinal model (TIM1) Funct. Foods Health Dis. 2014, 4, 222-231. DOI: 10.31989/ ffhd.v4i5.10. 